\title{
Immediate Effect of Calf Muscle Kinesio Taping on Ankle Joint Reposition Sense and Force Sense in Healthy Elderly
}

\author{
Jin-Tae Han \\ Department of Physical Therapy, College of Science, Kyungsung University, Busan, Republic of Korea
}

Purpose: The purpose of this study was to investigate the immediate effects of calf muscle Kinesio taping on ankle joint reposition sense (JRS) and force sense (FS) in healthy elderly.

Methods: Thirteen healthy elderly subjects were participated in this study. The error of ankle JRS and FS was evaluated by 3D motion capture device and digital dynamometer depending on three different taping conditions (Kinesio taping, sham taping, and no taping) respectively. All of subjects were asked to perform a proprioceptive task of ankle JRS and FS. One-way repeated ANOVA test was used to compare the error of JRS and FS depending on three different taping conditions.

Results: With Kinesio taping over calf muscle, ankle joint reposition sense error and force sense error significantly decreased, if compared with a sham taping or no taping condition.

Conclusion: To apply Kinesio taping over calf muscle could enhance ankle proprioceptive sense in the elderly people.

Keywords: Kinesio taping, Joint reposition sense, Force sense, Elderly

\section{INTRODUCTION}

Proprioception plays an essential role in activity of daily living. Impaired proprioception can cause a functional joint instability. ${ }^{1}$ Ankle proprioceptive sense is specially an important function in performance. ${ }^{2}$ Poor ankle proprioception have been identified as an important intrinsic factor to increase the risk of ankle injury. ${ }^{3}$

Ankle proprioception is critical to maintain the postural balance while performing functional activities such as standing, walking, and running. ${ }^{4}$ The proprioception includes the kinesthesia, joint reposition sense, and force sense. ${ }^{5}$ Ankle proprioception is most often evaluated by assessing ankle joint reposition sense and force sense. ${ }^{6}$ Proprioceptive ability declines with increasing age. This age-related decline in proprioception may aggravate postural balance control and increase falls risk in the elderly. ${ }^{7.8}$

A many studies have investigated the effect of interventions on ankle proprioceptive function to improve ankle instability. ${ }^{9,10}$ Recently, KinesioTaping (KT) has been extensively explored to enhance ankle proprioception in peoples with ankle instability. ${ }^{11,12}$ KT represents an interesting and

Received May 13, 2020 Revised Jul 10, 2020

Accepted Aug 12, 2020

Corresponding author Jin-Tae Han

E-mail jthan2001@ks.ac.kr relatively new intervention to treat musculoskeletal injuries. ${ }^{13}$

The continuous stretch applied on the skin under the adhesive taping might activate the mechanoreceptors, which in turn could stimulate a modulatory mechanism within the central nervous system and therefore increase muscular excitability. ${ }^{14}$

$\mathrm{KT}$ is an elastic tape that differs from the traditional non-elastic tape because of its ability to stretch up to $140 \%$ of its origin length, thereby providing a constant shear force on the skin. Kase et al $1{ }^{15}$ suggested that KT is expected the following results: it corrects muscle function by strengthening weak muscles, it improves blood and lymph circulation by eliminating tissue fluid or bleeding beneath the skin through muscle movement, it reduces pain through neurological suppression, it corrects misaligned joints by relieving muscle spasm.

However, several studies reported that application of KT could be useful for pain control ${ }^{16,17}$ and muscle function. ${ }^{18}$ Contrarily, a few studies found no difference muscle strength following the application of KT. ${ }^{19,20}$ There is limited scientific evidence to evaluate the KT effectiveness and the results are inconsistent and mixed. ${ }^{21}$

Copylight (C)2020 The Korean Society of Physical Therapy

This is an Open Access article distribute under the terms of the Creative Commons Attribution Non-commercial License (https:// creativecommons.org/license/by-nc/4.0.) which permits unrestricted non-commercial use, distribution, and reproduction in any medium, provided the original work is properly cited. 
Most of previous studies about ankle functions have usually examined the effect of taping in injured and uninjured young peoples. They suggested that current evidence about KT was not support the use of KT in musculoskeletal injury. ${ }^{22}$ However, there have no examined the effect of ankle taping on various fields such as proprioception, skin sensation et al. To evaluate the effect of KT need to test using diverse methods in clinical research. Then, it is necessary to investigate the effect of KT on proprioception using joint reposition sense and force sense in the old peoples.

The hypothesis of this study is that the application of KT could be decrease the ankle joint reposition sense error and force sense error in the elderly. Therefore, the purpose of this study is to investigate the effects of KT on ankle joint reposition sense and force sense in the elderly.

\section{METHODS}

\section{Subjects}

Thirteen healthy elderly subjects ( 8 males and 5 females) participated in this study, approved by the Human Research Ethics Committee of Kyungsung University-Protocol number KSU-19-03-003. After the purpose of the study was explained to subjects, they voluntarily agreed to participate this study and submitted their written informed consent. Healthy elderly who have not history of allergy to bandages were included in this study. The exclusion criteria were previous ankle fracture or sprain, calf muscle injuries and neurological problem within 6 months. ${ }^{13}$

\section{Procedures}

To measure the JRSE and FSE, subjects were seated on the table with the hip and knee placed at a $90^{\circ}$ position and the ankle in a neutral position without contacting floor. They were measured the ankle JRSE and FSE with Kinesio-taping (KT), sham-taping (ST), and no taping (NT) respectively. Subjects were barefoot during testing and were performed the task with their eyes closed and wore an eye patch. ${ }^{23}$ Randomized allocation was performed to assign subjects into KT, ST, and NT. All test was performed within 10 minutes to identify an immediate effect of three tape conditions on JRSE and FSE. To prevent the carry over effect, we examined the test with one tape condition a day for 3 days. A same tester measured the JRSE and the FSE.

\section{Kinesio taping application}

First taping condition was Kinesio taping. The calf muscle of the dominant leg was taped by an experienced physical therapist according to Ki- nesio-taping Manual. ${ }^{15}$ The skin was cleaned and shaved the calf muscle was stretched with the subject in the prone position. In this position, the tape of $5 \mathrm{~cm}$ width (BB tape, WETAPE Inc., Seoul, Korea) was applied from the proximal gastrocnemius muscle insertion to the calcaneus bone with $15-20 \%$ stretch tension. This tape strip was applied directly on the skin as follows 1) the tape was anchored at the heel with the ankle joint in a neutral position, 2) Calf muscle was stretched, 3) Y-type strip was divided proximal end of the tape and then were attached onto the medial and lateral heads of the gastrocnemius muscles, ${ }^{14} 4$ ) I-type strip was applied from the posterior surface of the calcaneus to the upper part of gastrocnemius junction (Figure 1A). ${ }^{15}$

Second taping condition was sham taping. The sham taping protocol consisted of placing three short strips of the same kind of material only onto the end of heel and medial/lateral heads of gastrocnemius muscles (Figure 1B). In this way, the sham taping was applied on the ineffective parts of the calf muscle without continuity, which is assumed not to have any effect. ${ }^{24}$ Third taping condition was no taping (Figure 1C).

\section{Measurement methods}

Measurements of the joint reposition sense error (JRSE) were taken using the 3D motion capture device (Myomotion Research PRO, Noraxon Inc., USA) that uses an inertial measurement unit sensor. To measure the JRSE, motion capture sensor was placed at the center of between 2nd and 3rd metatarsal bone. Subjects were performed passive ankle plantar flexion from staring position (ankle neutral position) to target angle (ankle plan$\operatorname{tar}$ flexion $20^{\circ}$ ). Subjects were asked to actively reproduce the target angles and were instructed to 'hold it there' for 5 seconds. The subjects were then asked to say 'now' when they perceived the ankle joint was at target angles.
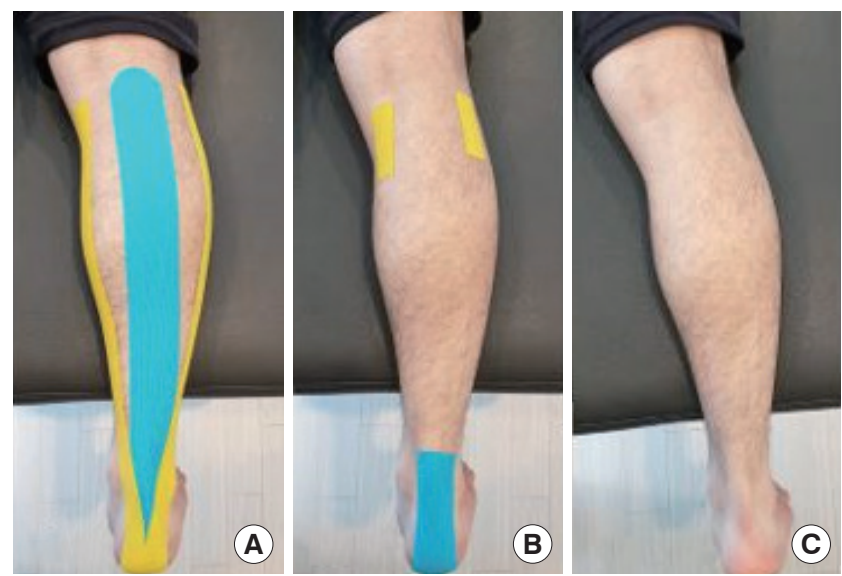

Figure 1. Three different taping conditions. (A): Kinesio taping, (B): Sham taping, (C): No taping. 
And then the reproduction angle of the ankle joint was recorded. All of tests were followed by a rest period in one minute (Figure 2A).

The force sense error (FSE) was measured by the Digital Dynamometer (PowerTrack II Commander, JTECH Medical, UT, USA). We first obtain a maximum force for ankle plantar flexion. Three trials were performed at target forces (50\% of maximum force). To measure the FSE, the subject performed ankle plantar flexion while receiving visual feedback. Once the subject achieved the target force, he or she was instructed to maintain it for 5 seconds. We then removed the visual feedback and instructed the subject to reproduce the target force. When the subject indicated verbally that he or she had achieved the target force, and we recorded it. We recorded the error value as the absolute difference between target force and the observed force for each trial (Figure 2B). All of test were measured three times in each condition.

\section{Statistical analysis}

The statistical analysis was performed using SPSS version 25 (SPSS Inc., Chicago, Illinois). Data normality was verified by means of the Shapiro-
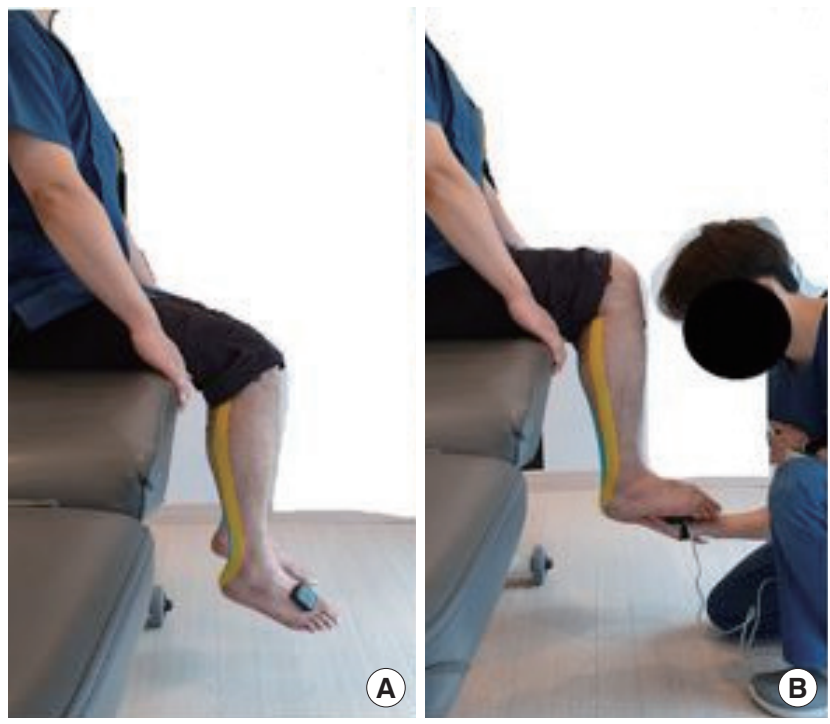

Figure 2. Measurement of ankle joint reposition sense and force sense. (A): Joint reposition sense, (B): Force sense.

Table 1. General characteristics

$($ Mean $\pm S D)$

\begin{tabular}{lc}
\hline & Subjects $(\mathrm{n}=13)$ \\
\hline Gender (male/female) & $8 / 5$ \\
K-MMSE (point) & $26.56 \pm 1.81$ \\
Age (yr) & $64.44 \pm 6.95$ \\
Height $(\mathrm{cm})$ & $167.33 \pm 7.02$ \\
Weight $(\mathrm{kg})$ & $67.67 \pm 6.10$ \\
\hline
\end{tabular}

K-MMSE: Korean version of Mini Mental State Examination.
Wilk test. We presented the mean value \pm standard deviation (SD) of each variable in all conditions (KT, ST and NT). We used One-way repeated ANOVA test to examine the effects of tape type and test of within-subjects contrasts to compare among three tape conditions respectively. The level of significance was set at $\mathrm{p}<0.05$.

\section{RESULTS}

\section{General characteristics of subjects}

Table 1 shows the general characteristics.

\section{Ankle joint reposition sense error}

Results of ankle JRSE are shown in Table 2. There are significant differences in ankle JRSE depending on taping conditions $(\mathrm{p}<0.05)$. Multiple comparisons test demonstrated that the mean of JRSE with KT was significantly lower than that of ST and NT (Table 2).

\section{Ankle force sense error}

Results of ankle FSE are shown in Table 3. There are significant differences in ankle FSE depending on taping conditions $(\mathrm{p}<0.05)$. Multiple comparisons test demonstrated that the mean of FSE with KT was significantly lower than that of ST and NT, and the FSE with ST was significantly lower than that of NT (Table 3).

\section{DISCUSSION}

Assessment of joint reposition sense and joint force sense is a highly reli-

Table 2. Comparison of ankle joint reposition sense error depending on taping conditions

(Unit: $\left.{ }^{\circ}\right)$

\begin{tabular}{|c|c|c|c|c|c|}
\hline \multirow{2}{*}{ Variable } & \multicolumn{3}{|c|}{ Taping conditions } & \multirow{2}{*}{$\mathrm{F}$} & \multirow{2}{*}{$p$} \\
\hline & $\mathrm{KT}$ & ST & NT & & \\
\hline JRSE & $5.56 \pm 1.09^{*}$ & $7.92 \pm 1.63$ & $9.96 \pm 2.57$ & 19.54 & $<0.01$ \\
\hline
\end{tabular}

JRSE: joint reposition sense error, KT: Kinesio taping, ST: Sham taping, NT: No taping. ${ }^{*} \mathrm{p}<0.05$ significantly difference between $\mathrm{KT}$ and NT.

Table 3. Comparison of ankle force sense error depending on taping conditions

(Unit: N)

\begin{tabular}{lccccc}
\hline \multirow{2}{*}{ Variable } & \multicolumn{3}{c}{ Taping conditions } & \multirow{2}{*}{ F } & p \\
\cline { 2 - 4 } & KT & ST & NT & & \\
\hline FSE & $5.37 \pm 1.39^{*+}$ & $8.09 \pm 2.21^{\neq}$ & $10.51 \pm 2.26$ & 27.96 & $<0.01$ \\
\hline
\end{tabular}

FSE: Force sense error, KT: Kinesio taping, ST: Sham taping, NT: No taping. ${ }^{*} \mathrm{p}<0.05$ : significantly difference between $\mathrm{KT}$ and $\mathrm{NT}$; ${ }^{+} \mathrm{p}<0.05$ : significantly difference between $\mathrm{KT}$ and $\mathrm{ST} ; \neq \mathrm{p}<0.05$ : significantly difference between $\mathrm{ST}$ and NT. 
able measure of proprioceptive sense. ${ }^{20}$ However, there was few studies to know simultaneously on the ankle joint reposition sense and force sense in the elderly. The purpose of the present study was to identify the immediately effect of KT on the proprioception sense in healthy elderly people. We investigated the difference of ankle joint reposition sense error and force sense error after applying with three different taping conditions. We added the I-type strip in KT condition to protect and support the gastrocnemius and soleus muscles during ankle movement.

The results of this study demonstrated that KT condition showed statistically significant improvements in ankle joint reposition sense error and ankle joint force sense error when compared to other conditions applying sham taping or no taping. Therefore, the findings of this study suggest that KT may be able to enhance joint reposition sense and joint force sense in healthy elderly. I considered that applying tape might affect the subject's proprioception sense similar to the previous studies. The results of this study agree with Seo et al. ${ }^{25}$ who evaluated the effect of KT on joint reposition sense of ankle. They suggested that KT improved the ankle joint reposition sense and $\mathrm{KT}$ is effective for the prevention of ankle sprain. I believed that KT elicits the proprioception sense through increased stimulation of cutaneous mechanoreceptors. ${ }^{26}$ Schultz et al. ${ }^{27}$ published the first detailed description of mechanoreceptors in human and suggested that they may have a proprioceptive function. Adachi et al. ${ }^{28}$ also reported that mechanoreceptors contribute to the joint position sense. Also, our finding agrees with Simmon et al. ${ }^{29}$ who suggested applying KT to subjects with ankle instability decreased force sense errors and KT may cause an increased awareness of muscular force. We believed these results suggested that KT can prevent muscle fatigue and help the learning effects and may affect the subject's psychological condition.

On the other hand, some studies reported that ankle taping had no effect on angle or force matching tasks. ${ }^{30,31}$ Parreira et al. ${ }^{32}$ reported that KT either provided no significant benefit, or its effect was too small to be clinically worthwhile. In future, studies should therefore include larger samples when assessing angle joint reposition sense or force sense.

Simon et al. ${ }^{28}$ reported that after KT application for an extended amount of time, the improvement of proprioceptive sense resulted in similar conscious in subjects with ankle instability. But Wison et al. ${ }^{33}$ reported that KT did not any significant differences in balance at the immediate and long-term application.

In other previous study, other intervention including tai chi exercise improved the proprioception at the ankle and knee joints in older people. ${ }^{34}$ Indeed, the large benefits of various intervention on proprioception may result in the maintenance of postural balance control in older people.

This study has several limitations; one of the limitations of this study is difficult to generalize because of the lack of subjects. In order to understand clearly the effects of KT on joint reposition sense and joint force sense, future study needs to be recruited the enough subjects. Another of limitations is that I assessed the short-term effects of KT, measured immediately after applying Kinesio tape. Some previous studies investigated the long-term effects related to the application of KT for as long as 1 or 2 days. ${ }^{17,35}$ Therefore, I suggest that future researcher should investigate not only the short-term effects but also long-term effects of KT.

In results, $\mathrm{KT}$ on the calf muscle show to enhance the joint reposition sense and force sense immediately after application in healthy elderly. Therefore, this study suggests that KT could be helpful intervention to improve the proprioceptive function in elderly people. Further research need to be conducted on other joints, on long-term effect of application of KT.

\section{ACKNOWLEDGEMENTS}

This research was supported by Kyungsung University Research Grants in 2019 .

\section{REFERENCES}

1. Han J, Waddington G, Anson J et al. Level of competitive success achieved by elite athletes and multi-joint proprioceptive ability. J Sci Med Sports. 2015;18(1):77-81.

2. Munn J, Sullivan SJ, Schneiders AG. Evidence of sensorimotor deficits in functional ankle instability: a systematic review with meta-analysis. J Sci Med Sports. 2010;13(1):2-12.

3. Witchalls J, Blanch P, Waddington G et al. Intrinsic functional deficits associated with increased risk of ankle injuries: a systematic review with meta-analysis. Br J Sports Med. 2012;46(7):515-23.

4. Lee AJY, Lin WH. Twelve-week biomechanical ankle platform system training on postural stability and ankle proprioception in subjects with unilateral functional ankle instability. Clin Biomech. 2008;23(8):106572 .

5. Riemann BL, Lephart SM. The sensorimotor system, part 1: the physiologic basis of functional joint stability. J Athl Train. 2002;37(1):71-9.

6. Hertel J. Sensorimotor deficits with ankle sprains and chronic ankle instability. Clin Sports Med. 2008;27(3):353-70.

7. Arvin M, Hoozemans MJ, Burger BJ et al. Reproducibility of a knee and hip proprioception test in healthy older adults. Aging Clin Exp Res. 2015;27 (2):171-7.

8. Westlake KP, Culham EG. Influence of testing position and age on measures of ankle proprioception. Adv Physiother. 2006;8(1):41-8.

9. Witchalls JB, Waddington G, Adams R et al. Chronic ankle instability af- 
fects learning rate during repeated proprioception testing. Phys Ther Sport. 2014;15(2):106-11.

10. Waddington G, Adams RD. The effect of a 5-week wobble-board exercise intervention on ability to discriminate different degrees of ankle inversion, barefoot and wearing shoes: a study in healthy elderly. J Am Geriatr Soc. 2004;52(4):573-6.

11. Hughes T, Rochester P. The effects of proprioceptive exercise and taping on proprioception in subjects with functional ankle instability: a review of the literature. Phys Ther Sport. 2008;9(3):136-47.

12. Refshauge KM, Kilbreath SL, Raymond J. The effect of recurrent ankle inversion sprain and taping on proprioception at the ankle. Med Sci Sports Exerc. 2000;32(1):10-5.

13. Fratocchi G, Mattia FD, Rossi R et al. Influence of Kinsio Taping applied over biceps brachii on isokinetic elbow peak torque. A placebo controlled study in a population of young healthy subjects. J Sci Med Sports. 2013;16(3):245-9.

14. Gomez-Soriano J, Abián-Vicén J, Aparicio-García C et al. The effects of kinesio taping on muscle tone in healthy subjects: a double-blind, placebo-controlled crossover trial. Man Ther. 2014;19(2):131-6.

15. Lee JH, Yoo WG. Treatment of chronic Achilles tendon pain by Kinesio taping in an amateur badminton player. Phys Ther Sport. 2012;15 (2):115-9.

16. Thelen MD, Dauber JA, Stoneman PD. The clinical efficacy of kinesio tape for shoulder pain: a randomized, double-blinded, clinical trial. J Orthop Sports Phys Ther. 2008;38(7):389-95.

17. González-Iglesias J, Fernández-de-Las-Peñas C, Cleland JA et al. Shortterm effects of cervical Kinesio Taping on pain and cervical range of motion in patients with acute whiplash injury: a randomized clinical trial. J Orthop Sports Phys Ther. 2009;39(7):515-21.

18. Slupik A, Dwornik M, Bialoszewski D et al. Effect of Kinsio Taping on bioelectrical activity of vastus medialis muscle. Orthop Traumatol Rehabil. 2007;9(6):644-51.

19. Fu TC, Wong AM, Pei YC et al. Effect of Kinesio Taping on muscle strength in athletes-a pilot study. J Sci Med Sports. 2008;11(2):198-201.

20. Chang HY, Chou KY, Lin JJ et al. Immediate effect of forearm Kinesio Taping on maximal grip strength and force sense in healthy collegiate athlete. Phys Ther Sport. 2010;11(4):122-7.

21. Williams S, Whatman C, Hume PA et al. Kinesio taping in treatment and prevention of sports injury: A meta-analysis of the evidence for its effectiveness. Sports Medicine. 2012;42:153-64

22. Parreira P do CS, Costa L da CM, Hespanhol Junior LC et al. Current evidence does not support the use of Kinesio Taping in clinical practice: a systematic review. J Physiother. 2014;60(1):31-9.

23. Miura K, Ishibashi Y, Tsuda E et al. The effect of local and general fatigue on knee proprioception. Arthroscopy. 2004;20(4):414-8.

24. Karadag-Saygi E, Cubykcu-Aydoseli K, Kablan N et al. The role of kinesiotaping combined with botulinum toxin to reduce plantar flexors spasticity after stroke. Top Stroke Rehabil. 2010;17(4):318-22.

25. Seo HD, Kim MY, Choi JE et al. Effects of Kinesio taping on joint position sense of the ankle. J Phys Ther Sci. 2016;28(4):1158-60.

26 Liu K, Qian J, Gao Z et al. Effects of Kinesio taping of the knee on proprioception, balance, and functional performance in patients with anterior cruciate ligament rupture: A retrospective case series. Medicine. 2019;39(48):e17956.

27. Schultz RA, Miller DC, Kerr CS et al. Mechanoreceptors in human cruciate ligaments: a histologic study. J Bone Joint Surg. 1984;66(7):1072-6.

28. Adachi N, Ochi M, Uchio Y et al. Mechanoreceptors in the anterior cruciate ligament contribute to the joint position sense. Acta Orthop Scand. 2002;73(3):330-4.

29. Simmon J, Garcia W, Docherty CL. The effect of kinesio tape on force sense in people with functional ankle instability. Clin J Sport Med. 2014;24(4):289-94.

30. Halseth T, McChesney JW, DeBeliso M et al. The effects of kinesio ${ }^{\mathrm{TM}}$ taping on proprioception at the ankle. J Sports Sci Med. 2004;3(1):1-7.

31. Hopper DM, Grisbrook TL, Finucane M et al. Effect of ankle taping on angle and force matching and strength of the plantar flexors. Phys Ther Sport. 2014;15(4):254-60.

32. Parreira Pdo CSP, Costa Lda CM, Hespanhol Junior LC et al. Current evidence does not support the use of Kinesio Taping in clinical practice: a systematic review. J Phyiother. 2014;60(1):31-9.

33. Wison V, Douris P, Fukunroku T et al. The immediate and long-term effects of kinesiotape on balance and functional performance. Int J Sports Phys Ther. 2016;11(2):247-53.

34. Xu D, Hong Y, Li J et al. Effect of tai chi exercise on proprioception of ankle and knee joint in old people. Br J Sports Med. 2004;38(1):50-4.

35. Park SJ, Youn PS. The immediate effect of wrist joint mobilization with taping on range of motion, grip strength, spasticity in stroke patients. J Kor Phys Ther. 2017;29(4):187-93. 